\title{
Application of Tanh Method to Complex Coupled Nonlinear Evolution Equations
}

\author{
M.A. AbDelkaWY ${ }^{a, b}$, A.H. BhraWy ${ }^{b}$, E. Zerrad ${ }^{c}$ And A. Biswas ${ }^{d, e}$ \\ ${ }^{a}$ Department of Mathematics and Statistics, College of Science, Al-Imam Mohammad Ibn Saud Islamic University, \\ Riyadh-11566, Saudi Arabia \\ ${ }^{b}$ Department of Mathematics, Faculty of Science, Beni-Suef University, Beni-Suef, Egypt \\ ${ }^{c}$ Department of Physics and Engineering, Delaware State University, Dover, DE 19901-2277, USA \\ ${ }^{d}$ Department of Mathematical Sciences, Delaware State University, Dover, DE 19901-2277, USA \\ ${ }^{e}$ Department of Mathematics, Faculty of Science, King Abdulaziz University, Jeddah-21589, Saudi Arabia
}

(Received June 27, 2015; in final form February 28, 2016)

\begin{abstract}
This paper studies the application of tanh method to address a few coupled nonlinear evolution equations that are in complex domain. There are soliton solutions as well as triangular solutions that are revealed with this integration scheme. The equations studied in this paper are applicable to various branches of applied and theoretical physics.
\end{abstract}

DOI: 10.12693/APhysPolA.129.278

PACS/topics: 02.30.Ik, 02.30.Jr, 05.45.Yv

\section{Introduction}

The theory of nonlinear evolution equations (NLEEs) is one of the key issues in applied and theoretical physics as well as in engineering sciences and applied mathematics. These NLEEs appear in fluid dynamics, nonlinear optics, plasma physics, nuclear physics, mathematical biosciences, and several others [1-11]. Integrability aspect is one of the main focus areas of research for these NLEEs. There are several integration tools that are proposed and successfully applied to handle NLEEs during the past couple of decades. Some of these tools are Adomian decomposition method, $G^{\prime} / G$-expansion method, exp-method, the Lie symmetry analysis, the Riccati equation approach, simplest equation method, the Kudryashov method and several others $[4,6,7,9]$. These techniques are well beyond the classical inverse scattering transform (IST) that has very limited applications [12-14]. Once upon a time, IST was a monopoly to study NLEEs and it was applicable only to those NLEEs which passed the Painleve test of integrability. However, these current tools are applicable even to equations which fail the Painleve test. Thanks to these modern methods of integrability that widens the scope of integration for such NLEEs.

While this paper addresses complex-valued NLEEs, there are several real-valued NLEEs that exist in the literature. Those results are reported and have exhausted the literature. Some of these are the well known Korteweg-de Vries (KdV) equation, the Boussinesq equation, the Kawahara equation, the BenjaminBona-Mahoney equation, the Peregrine equation, regularized long wave (RLW) equation and others. These models study shallow water wave flow along lake-shores and sea beaches. Besides them, there are additional models in real domain that describe these features such as the Rosenau-KdV equation, the Rosenau-Kawahara equation, the Rosenau-KdV-RLW equation. These model dispersive shallow water wave dynamics. Finally, coupled NLEEs such as the Bona-Chen equation and GearGrimshaw model study two-layered shallow water waves.

This paper focuses on vector coupled complex-valued NLEEs that are applicable in several physical situations. One such model is the Davey-Stewartson equation that describes the evolution of three-dimensional wave packet on water of finite depth. Another one is vector coupled nonlinear Schrödinger's equation that is studied in birefringent fibers, optical couplers as well as in dense wavelength division multiplexed (DWDM) system. This paper will, however, focus on the Schrödinger-Boussinesq equation, the Higgs field equation and the Maccari equation. The following subsection introduces these models in a very succinct manner. Subsequently the integration algorithm, employed in this paper, will be reviewed and finally it will be applied to the three models.

\subsection{Complex-valued coupled NLEEs}

The generalized complex coupled SchrödingerBoussinesq equations study the stationary propagation of coupled nonlinear upper-hybrid and magnetosonic waves in magnetized plasmas [15-21]. The complex coupled generalized Schrödinger-Boussinesq equation can be written as

$$
\begin{aligned}
& \mathrm{i} u_{t}+u_{x x}+\alpha u=u v, \\
& 3 v_{t t}-v_{x x x x}+3\left(v^{2}\right)_{x x}+\beta v_{x x}=\left(|u|^{2}\right)_{x x} .
\end{aligned}
$$

The complex coupled Higgs field equation introduces quantum field (or the Higgs field) to illustrate the generation mechanism of mass for gauge bosons $[15,21]$ can be written in the form

$$
\begin{aligned}
& u_{t t}-u_{x x}-\alpha u+\beta|u|^{2} u-2 u v=0 \\
& v_{t t}+v_{x x}-\beta\left(|u|^{2}\right)_{x x}=0,
\end{aligned}
$$

where $v$ and $u$ represent complex scalar nucleon field and scalar meson field, respectively. 
The complex coupled Maccari equations is a nonlinear evolution equations described the motion of the isolated waves, localized in a small part of space, in many fields such as hydrodynamic, plasma physics, nonlinear optics and others [22-28]. Complex coupled Maccari equation derived from the Kadomtsev-Petviashvili equation (the best known two-dimensional generalizations of the $\mathrm{KdV}$ equation) and can be written in the form

$$
\begin{aligned}
& \mathrm{i} u_{t}+u_{x x}+u v=0, \\
& v_{t}+v_{y}+\left(|u|^{2}\right)_{x}=0,
\end{aligned}
$$

which is called integrable $(2+1)$-dimensional nonlinear Maccari system [23].

\section{Recapitulation of generalized tanh method}

In this section, the method will be described briefly. Consider the given NLEEs

$$
G\left(u, u_{x}, u_{y}, u_{t}, u_{x y}, \ldots\right)=0 .
$$

The first step is to unite the independent variables $x, y$ and $t$ into one particular variable through the definition

$$
\zeta=x+\alpha y+\beta t, \quad u(x, y, t)=U(\zeta),
$$

and Eq. (4) will be changed into the ordinary differential equation (ODE):

$$
G\left(U, U^{\prime}, U^{\prime \prime}, U^{\prime \prime \prime}, \ldots\right)=0 .
$$

Our aim is to obtain exact solutions, if possible, for this ODE. To this end, we propose a new variable

$$
\psi=\psi(\zeta)
$$

which is an exact solution of the Riccati equation

$$
\psi^{\prime}=k+\psi^{2} \text {. }
$$

Let the following series expansion is a solution of Eq. (4):

$$
u(x, y, t)=U(\zeta)=\sum_{i=0}^{m} a_{i} \psi^{i} .
$$

We can evaluate $m$ by balancing the highest order of the linear term(s) with highest order of the nonlinear one(s). Normally $m$ is a positive integer, so that an analytic solution in closed form may be obtained. Substituting (6) into (7) and comparing the coefficients of each power of $\psi$ in both sides, an over-determined system of nonlinear algebraic equations in terms of to $k, a_{0}, a_{1} \ldots$ is obtained. One may find the solution of this over-determined system e.g. by means of symbolic computation program, say MATHEMATICA. We can obtain several exact solutions:

(i) for $k<0$

$$
\psi=\left\{\begin{array}{l}
-\sqrt{-k} \operatorname{coth}(\sqrt{-k} \zeta), \\
-\sqrt{-k} \tanh (\sqrt{-k} \zeta),
\end{array}\right.
$$

(ii) for $k=0$

$$
\psi=\frac{-1}{\zeta+c},
$$

(iii) for $k>0$

$$
\psi=\left\{\begin{array}{l}
-\sqrt{k} \cot (\sqrt{k} \zeta), \\
\sqrt{k} \tan (\sqrt{k} \zeta) .
\end{array}\right.
$$

Another advantage of the Riccati Eq. (6) is that the sign of $k$ can be used to exactly judge the amount and types of traveling wave solution of Eq. (4).

\section{Applications}

Applications of this integration algorithm is widespread as seen in various journals and books. This paper will apply the tool to a few specific type of NLEEs. These are complex-valued coupled NLEEs which are applicable to various areas of applied and theoretical physics. The detailed discussions are in the next three subsections.

\subsection{Higgs field equation}

We first consider the complex coupled Higgs field equation

$$
\begin{aligned}
& u_{t t}-u_{x x}-\alpha u+\beta|u|^{2} u-2 u v=0, \\
& v_{t t}+v_{x x}-\beta\left(|u|^{2}\right)_{x x}=0,
\end{aligned}
$$

using the transformations

$$
\begin{aligned}
& u(x, t)=\mathrm{e}^{\mathrm{i} \theta} U(\zeta), \quad v(x, t)=V(\zeta), \\
& \zeta=x+\nu t, \quad \theta=p x+r t,
\end{aligned}
$$

we obtain the relation $p=r \nu$ and carry Eq. (11) into the system of ordinary differential equations (SODEs):

$$
\begin{aligned}
& \left(\nu^{2}-1\right) U^{\prime \prime}+\left(p^{2}-r^{2}-\alpha\right) U+\beta U^{3}-2 U V=0, \\
& \left(\nu^{2}+1\right) V^{\prime \prime}-2 \beta\left(U^{\prime}\right)^{2}-2 \beta U U^{\prime \prime}=0,
\end{aligned}
$$

integrating the second equation in Eq. (11) twice gives

$$
\left(\nu^{2}+1\right) V=\beta U^{2} .
$$

Using Eq. (14) into Eq. (11), yields only one ODE:

$$
\begin{aligned}
& \left(\nu^{2}-1\right)\left(\nu^{2}+1\right) U^{\prime \prime}+\left(p^{2}-r^{2}-\alpha\right) U \\
& \quad+\beta\left(\nu^{2}-1\right) U^{3}=0 .
\end{aligned}
$$

Balancing the term $U^{\prime \prime}$ with the term $U^{3}$ we obtain $m=1$ then

$$
U(\zeta)=\sum_{i=0}^{1} a_{i} \psi^{i}=a_{0}+a_{1} \psi,
$$

substituting Eq. (16) into Eq. (15) and comparing the coefficients of each power of $\psi$ in both sides, we get an overdetermined system of nonlinear algebraic equations with respect to $\nu, a_{i} ; i=0,1$. Solving the over-determined system of nonlinear algebraic equations using of $M A T H$ EMATICA, we obtain

$$
\begin{aligned}
& a_{0}=0, \quad a_{1}= \pm \mathrm{i} \sqrt{2\left(1+\nu^{2}\right) / \beta} \\
& \text { and } k=\frac{r^{2}\left(1-\nu^{2}\right)+\alpha}{2\left(\nu^{2}-1\right)},
\end{aligned}
$$

we find the following solutions of Eq. (15):

[i.] for $k<0$

$$
\begin{aligned}
& U_{1,2}= \pm \mathrm{i} \sqrt{\frac{2\left(1+\nu^{2}\right)\left(r^{2}\left(1-\nu^{2}\right)+\alpha\right)}{2 \beta\left(1-\nu^{2}\right)}} \\
& \quad \times \operatorname{coth}\left(\sqrt{\frac{r^{2}\left(1-\nu^{2}\right)+\alpha}{2\left(1-\nu^{2}\right)}} \zeta\right),
\end{aligned}
$$




$$
\begin{aligned}
& U_{3,4}= \pm \mathrm{i} \sqrt{\frac{2\left(1+\nu^{2}\right)\left(r^{2}\left(1-\nu^{2}\right)+\alpha\right)}{2 \beta\left(1-\nu^{2}\right)}} \\
& \times \tanh \left(\sqrt{\frac{r^{2}\left(1-\nu^{2}\right)+\alpha}{2\left(1-\nu^{2}\right)} \zeta}\right),
\end{aligned}
$$

[ii.] for $k=0$

$$
U_{5,6}=\frac{ \pm \mathrm{i} \sqrt{2\left(1+\nu^{2}\right) / \beta}}{\zeta},
$$

[iii.] for $k>0$

$$
\begin{aligned}
& U_{7,8}= \pm \mathrm{i} \sqrt{\frac{2\left(1+\nu^{2}\right)\left(r^{2}\left(1-\nu^{2}\right)+\alpha\right)}{2 \beta\left(\nu^{2}-1\right)}} \\
& \times \cot \left(\sqrt{\left.\frac{r^{2}\left(1-\nu^{2}\right)+\alpha}{2\left(\nu^{2}-1\right)} \zeta\right),}\right. \\
& U_{9,10}= \pm \mathrm{i} \sqrt{\frac{2\left(1+\nu^{2}\right)\left(r^{2}\left(1-\nu^{2}\right)+\alpha\right)}{2 \beta\left(\nu^{2}-1\right)}} \\
& \times \tan \left(\sqrt{\frac{r^{2}\left(1-\nu^{2}\right)+\alpha}{2\left(\nu^{2}-1\right)}} \zeta\right) .
\end{aligned}
$$

Then the solutions of the complex coupled Higgs field Eq. (11) are

$$
\begin{aligned}
& u_{1,2}= \pm \mathrm{i} \mathrm{e}^{\mathrm{i}(r \nu x+r t)} \sqrt{\frac{2\left(1+\nu^{2}\right)\left(r^{2}\left(1-\nu^{2}\right)+\alpha\right)}{2 \beta\left(1-\nu^{2}\right)}} \\
& \times \operatorname{coth}\left(\sqrt{\frac{r^{2}\left(1-\nu^{2}\right)+\alpha}{2\left(1-\nu^{2}\right)}}(x+\nu t)\right) \text {, } \\
& v_{1,2}=\frac{2\left(1+\nu^{2}\right)\left(r^{2}\left(1-\nu^{2}\right)+\alpha\right)}{2 \beta\left(\nu^{2}-1\right)} \\
& \times \operatorname{coth}^{2}\left(\sqrt{\frac{r^{2}\left(1-\nu^{2}\right)+\alpha}{2\left(1-\nu^{2}\right)}}(x+\nu t)\right), \\
& u_{3,4}= \pm \mathrm{i} \mathrm{e}^{\mathrm{i}(r \nu x+r t)} \sqrt{\frac{2\left(1+\nu^{2}\right)\left(r^{2}\left(1-\nu^{2}\right)+\alpha\right)}{2 \beta\left(1-\nu^{2}\right)}} \\
& \times \tanh \left(\sqrt{\frac{r^{2}\left(1-\nu^{2}\right)+\alpha}{2\left(1-\nu^{2}\right)}}(x+\nu t)\right), \\
& v_{3,4}=\frac{2\left(1+\nu^{2}\right)\left(r^{2}\left(1-\nu^{2}\right)+\alpha\right)}{2 \beta\left(\nu^{2}-1\right)} \\
& \times \tanh ^{2}\left(\sqrt{\frac{r^{2}\left(1-\nu^{2}\right)+\alpha}{2\left(1-\nu^{2}\right)}}(x+\nu t)\right), \\
& u_{5,6}=\frac{ \pm \mathrm{i} \mathrm{e}^{\mathrm{i}(r \nu x+r t)} \sqrt{2\left(1+\nu^{2}\right) / \beta}}{(x+\nu t)}, \\
& v_{5,6}=-\frac{2\left(1+\nu^{2}\right)}{\beta(x+\nu t)^{2}},
\end{aligned}
$$

$$
\begin{aligned}
& u_{7,8}= \pm \mathrm{i} \mathrm{e}^{\mathrm{i}(r \nu x+r t)} \sqrt{\frac{2\left(1+\nu^{2}\right)\left(r^{2}\left(1-\nu^{2}\right)+\alpha\right)}{2 \beta\left(\nu^{2}-1\right)}} \\
& \times \cot \left(\sqrt{\frac{r^{2}\left(1-\nu^{2}\right)+\alpha}{2\left(\nu^{2}-1\right)}}(x+\nu t)\right), \\
& v_{7,8}=\frac{2\left(1+\nu^{2}\right)\left(r^{2}\left(1-\nu^{2}\right)+\alpha\right)}{2 \beta\left(1-\nu^{2}\right)} \\
& \times \cot ^{2}\left(\sqrt{\frac{r^{2}\left(1-\nu^{2}\right)+\alpha}{2\left(\nu^{2}-1\right)}}(x+\nu t)\right), \\
& u_{9,10}= \pm \mathrm{i} \mathrm{e}^{\mathrm{i}(r \nu x+r t)} \sqrt{\frac{2\left(1+\nu^{2}\right)\left(r^{2}\left(1-\nu^{2}\right)+\alpha\right)}{2 \beta\left(\nu^{2}-1\right)}} \\
& \times \tan \left(\sqrt{\frac{r^{2}\left(1-\nu^{2}\right)+\alpha}{2\left(\nu^{2}-1\right)}}(x+\nu t)\right), \\
& u_{9,10}=\frac{2\left(1+\nu^{2}\right)\left(r^{2}\left(1-\nu^{2}\right)+\alpha\right)}{2 \beta\left(1-\nu^{2}\right)} \\
& \times \tan ^{2}\left(\sqrt{\frac{r^{2}\left(1-\nu^{2}\right)+\alpha}{2\left(\nu^{2}-1\right)}}(x+\nu t)\right) .
\end{aligned}
$$

\subsection{Schrödinger-Boussinesq equation}

In this subsection, we present exact solutions for complex coupled generalized Schrödinger-Boussinesq equation

$$
\begin{aligned}
& \mathrm{i} u_{t}+u_{x x}+\alpha u=u v, \\
& 3 v_{t t}-v_{x x x x}+3\left(v^{2}\right)_{x x}+\beta v_{x x}=\left(|u|^{2}\right)_{x x},
\end{aligned}
$$

using the transformation

$$
u(x, t)=\mathrm{e}^{\mathrm{i} \theta} U(\zeta), \quad \zeta=x+\nu t, \quad \theta=p x+r t,
$$

then we obtain the relation $\nu=-2 p$ and carry Eq. (26) into the SODEs

$$
\begin{aligned}
& \left(\alpha-r-p^{2}\right) U+U^{\prime \prime}-U V=0, \\
& \left(\beta+12 p^{2}\right) V^{\prime \prime}+\left(3 V^{2}-U^{2}\right)^{\prime \prime}-V^{(4)}=0,
\end{aligned}
$$

integrate the second equation in Eq. (28) twice we obtain

$$
\begin{aligned}
& \left(\alpha-r-p^{2}\right) U+U^{\prime \prime}-U V=0, \\
& \left(\beta+12 p^{2}\right) V+\left(3 V^{2}-U^{2}\right)-V^{\prime \prime}=0 .
\end{aligned}
$$

Using the techniques in Sect. 2, we find

$$
\begin{aligned}
& U(\zeta)=\sum_{2=0}^{2} a_{i} \psi^{i}=a_{0}+a_{1} \psi+a_{2} \psi^{2}, \\
& V(\zeta)=\sum_{i=0}^{2} b_{i} \psi^{i}=b_{0}+b_{1} \psi+b_{2} \psi^{2} .
\end{aligned}
$$

Proceeding as in the previous case we obtain different four cases: 
[i.]

$$
\begin{aligned}
& a_{1}=b_{1}=0, \quad b_{0}=\frac{3}{22}(12 \alpha-12 r+\beta), \\
& k=\frac{1}{44}(12 \alpha-12 r+\beta), \quad a_{0}=\frac{36 \alpha-36 r+3 \beta}{11 \sqrt{2}}, \\
& a_{2}=6 \sqrt{2}, \quad b_{2}=6 \text { and } p=\sqrt{\frac{r-\alpha-\beta}{11}} .
\end{aligned}
$$

[ii.]

$$
\begin{aligned}
& a_{1}=b_{1}=0, \quad b_{0}=\frac{7}{54}(12 \alpha-12 r+\beta), \\
& k=\frac{1}{36}(12 \alpha-12 r+\beta), \quad a_{0}=\frac{12 \alpha-12 r+\beta}{3 \sqrt{2}} \\
& a_{2}= \pm 6 \sqrt{2}, \quad b_{2}=6 \text { and } p= \pm \frac{1}{3} \sqrt{\alpha-r-2 \beta / 3}
\end{aligned}
$$

[iii.]

$$
\begin{aligned}
& a_{1}=b_{1}=0, \quad b_{0}=\frac{-1}{22}(12 \alpha-12 r+\beta), \\
& k=\frac{-1}{44}(12 \alpha-12 r+\beta), a_{0}=\frac{12 r-12 \alpha-\beta}{11 \sqrt{2}}, \\
& a_{2}=6 \sqrt{2}, b_{2}=6 \text { and } p=\sqrt{\frac{r-\alpha-\beta}{11}} .
\end{aligned}
$$

[iv.]

$$
\begin{aligned}
& a_{1}=b_{1}=0, \quad b_{0}=\frac{5}{54}(12 r-12 \alpha-\beta), \\
& k=\frac{1}{36}(12 r-12 \alpha-\beta), \quad a_{0}=\frac{12 \alpha-12 r+\beta}{9 \sqrt{2}}, \\
& a_{2}= \pm 6 \sqrt{2}, \quad b_{2}=6 \text { and } p= \pm \frac{1}{3} \sqrt{\frac{r-\alpha-2 \beta / 3}{11}}
\end{aligned}
$$

From the first case we find five solutions of the complex coupled generalized Schrödinger-Boussinesq system Eq. (26) are

$$
\begin{aligned}
u_{1} & =\mathrm{e}^{\mathrm{i}(\sqrt{(r-\alpha-\beta) / 11} x+r t)}\left[\frac{36 \alpha-36 r+3 \beta}{11 \sqrt{2}}\right. \\
& -6 \sqrt{\frac{12 r-12 \alpha-\beta}{22}} \operatorname{coth}\left(\sqrt{\frac{12 r-12 \alpha-\beta}{44}}\right. \\
& \left.\left.\times\left(x-2 \sqrt{\frac{r-\alpha-\beta}{11}} t\right)\right)\right], \\
v_{1} & =\frac{36 \alpha-36 r+3 \beta}{22}-6 \sqrt{\frac{12 r-12 \alpha-\beta}{444}} \\
& \times \operatorname{coth}\left(\sqrt{\frac{12 r-12 \alpha-\beta}{44}}\left(x-2 \sqrt{\frac{r-\alpha-\beta}{11}} t\right)\right), \\
u_{2} & =\mathrm{e}^{\mathrm{i}(\sqrt{(r-\alpha-\beta) / 11} x+r t)}\left[\frac{36 \alpha-36 r+3 \beta}{11 \sqrt{2}}\right. \\
-6 & \sqrt{\frac{12 r-12 \alpha-\beta}{22}} \tanh \left(\sqrt{\frac{12 r-12 \alpha-\beta}{44}}\right.
\end{aligned}
$$

$$
\begin{aligned}
& \left.\left.\times\left(x-2 \sqrt{\frac{r-\alpha-\beta}{11}} t\right)\right)\right], \\
v_{2} & =\frac{36 \alpha-36 r+3 \beta}{22}-6 \sqrt{\frac{12 r-12 \alpha-\beta}{444}} \\
& \times \tanh \left(\sqrt{\frac{12 r-12 \alpha-\beta}{44}}\left(x-2 \sqrt{\frac{r-\alpha-\beta}{11}} t\right)\right),
\end{aligned}
$$$$
u_{3}=\mathrm{e}^{\mathrm{i}(\sqrt{(r-\alpha-\beta) / 11} x+r t)}\left[\frac{36 \alpha-36 r+3 \beta}{11 \sqrt{2}}\right.
$$$$
\left.-\frac{6 \sqrt{2}}{x-2 \sqrt{(r-\alpha-\beta) / 11} t}\right] \text {, }
$$$$
v_{3}=\frac{36 \alpha-36 r+3 \beta}{22}-\frac{6}{x-2 \sqrt{(r-\alpha-\beta) / 11} t},
$$$$
u_{4}=\mathrm{e}^{\mathrm{i}(\sqrt{(r-\alpha-\beta) / 11} x+r t)}\left[\frac{36 \alpha-36 r+3 \beta}{11 \sqrt{2}}\right.
$$$$
-6 \sqrt{\frac{12 \alpha-12 r+\beta}{22}} \cot \left(\sqrt{\frac{12 \alpha-12 r+\beta}{44}}\right.
$$$$
\left.\times\left(x-2 \sqrt{\frac{r-\alpha-\beta}{11}} t\right)\right),
$$$$
v_{4}=\frac{36 \alpha-36 r+3 \beta}{22}-6 \sqrt{\frac{12 \alpha-12 r+\beta}{44}}
$$$$
\times \cot \left(\sqrt{\frac{12 \alpha-12 r+\beta}{44}}\left(x-2 \sqrt{\frac{r-\alpha-\beta}{11}} t\right)\right),
$$$$
u_{5}=\mathrm{e}^{\mathrm{i}(\sqrt{(r-\alpha-\beta) / 11} x+r t)}\left[\frac{36 \alpha-36 r+3 \beta}{11 \sqrt{2}}\right.
$$

$$
\begin{aligned}
& +6 \sqrt{\frac{12 \alpha-12 r+\beta}{22}} \tan \left(\sqrt{\frac{12 \alpha-12 r+\beta}{44}}\right. \\
& \left.\left.\times\left(x-2 \sqrt{\frac{r-\alpha-\beta}{11}} t\right)\right)\right], \\
& v_{5}=\frac{36 \alpha-36 r+3 \beta}{22}+6 \sqrt{\frac{12 \alpha-12 r+\beta}{44}} \\
& \times \tan \left(\sqrt{\frac{12 \alpha-12 r+\beta}{44}}\left(x-2 \sqrt{\frac{r-\alpha-\beta}{11}} t\right)\right) .
\end{aligned}
$$

The last three above cases given other fifteen solutions.

\subsection{Maccari equation}

The $(2+1)$-dimensional nonlinear complex coupled Maccari equations are the second complex coupled equations that will be discussed

$$
\mathrm{i} u_{t}+u_{x x}+u v=0, \quad v_{t}+v_{y}+\left(|u|^{2}\right)_{x}=0,
$$




$$
\begin{aligned}
& u(x, t)=\mathrm{e}^{\mathrm{i} \theta} U(\zeta), \quad v(x, y, t)=V(\zeta), \\
& \zeta=x+y+\nu t, \quad \theta=p x+q y+r t,
\end{aligned}
$$

we obtain the relation $\nu=-2 p$ and carry Eq. (40) into the SODEs

$$
\begin{aligned}
& \left(p^{2}+r\right) U-U^{\prime \prime}-U V=0, \\
& (\nu+1) V^{\prime}+2 U U^{\prime}=0,
\end{aligned}
$$

if we integrate the second equation in Eq. (42) once, we obtain

$$
(\nu+1) V=U^{2},
$$

using Eq. (42) into (40), we obtain the following ODE:

$$
(1-2 p)\left(p^{2}+r\right) U-(1-2 p) U^{\prime \prime}+U^{3}=0 .
$$

Based on the information included in Sect. 2, we obtain

$$
\begin{aligned}
& a_{0}=0, \quad a_{1}= \pm \sqrt{2-4 p}, \\
& \text { and } k=\frac{1+\left(p^{2}+r\right)(1-2 p)}{2-4 p},
\end{aligned}
$$

consequently, one yields the solutions of Eq. (44): [i.] for $k<0$

$$
\begin{aligned}
& U_{1,2}= \pm \mathrm{i} \sqrt{1+\left(p^{2}+r\right)(1-2 p)} \\
& \times \operatorname{coth}\left(\sqrt{\frac{1+\left(p^{2}+r\right)(1-2 p)}{4 p-2}} \zeta\right), \\
& U_{3,4}= \pm \mathrm{i} \sqrt{1+\left(p^{2}+r\right)(1-2 p)} \\
& \quad \times \tanh \left(\sqrt{\frac{1+\left(p^{2}+r\right)(1-2 p)}{4 p-2}} \zeta\right),
\end{aligned}
$$

[ii.] for $k=0$

$$
U_{5,6}=\frac{ \pm \sqrt{2-4 p}}{\zeta},
$$

[iii.] for $k>0$

$$
\begin{aligned}
& U_{7,8}= \pm \sqrt{1+\left(p^{2}+r\right)(1-2 p)} \\
& \times \cot \left(\sqrt{\frac{1+\left(p^{2}+r\right)(1-2 p)}{2-4 p} \zeta}\right), \\
& U_{9,10}= \pm \sqrt{1+\left(p^{2}+r\right)(1-2 p)} \\
& \times \tan \left(\sqrt{\frac{1+\left(p^{2}+r\right)(1-2 p)}{2-4 p}} \zeta\right) .
\end{aligned}
$$

Then the solutions of $(2+1)$-dimensional nonlinear complex coupled Maccari Eq. (40) are

$$
\begin{aligned}
& u_{1,2}= \pm \mathrm{i}^{\mathrm{i}(p x+q y+r t)} \sqrt{1+\left(p^{2}+r\right)(1-2 p)} \\
& \times \operatorname{coth}\left(\sqrt{\frac{1+\left(p^{2}+r\right)(1-2 p)}{4 p-2}}(x+y-2 p t)\right), \\
& v_{1,2}=\frac{1+\left(p^{2}+r\right)(1-2 p)}{1-2 p}
\end{aligned}
$$

$$
\begin{aligned}
& \times \operatorname{coth}^{2}\left(\sqrt{\frac{1+\left(p^{2}+r\right)(1-2 p)}{4 p-2}}(x+y-2 p t)\right), \\
& u_{3,4}= \pm \mathrm{i} \mathrm{e}^{\mathrm{i}(p x+q y+r t)} \sqrt{1+\left(p^{2}+r\right)(1-2 p)} \\
& \times \tanh \left(\sqrt{\frac{1+\left(p^{2}+r\right)(1-2 p)}{4 p-2}}(x+y-2 p t)\right), \\
& v_{3,4}=\frac{1+\left(p^{2}+r\right)(1-2 p)}{1-2 p} \\
& \times \tanh ^{2}\left(\sqrt{\frac{1+\left(p^{2}+r\right)(1-2 p)}{4 p-2}}(x+y-2 p t)\right) \\
& u_{5,6}=\frac{ \pm \mathrm{e}^{\mathrm{i}(p x+q y+r t)} \sqrt{2-4 p}}{(x+y-2 p t)} \text {, } \\
& v_{5,6}=\frac{-2}{(x+y-2 p t)^{2}}, \\
& u_{7,8}= \pm \mathrm{e}^{\mathrm{i}(p x+q y+r t)} \sqrt{1+\left(p^{2}+r\right)(1-2 p)} \\
& \times \cot \left(\sqrt{\frac{1+\left(p^{2}+r\right)(1-2 p)}{2-4 p}}(x+y-2 p t)\right), \\
& v_{7,8}=\frac{1+\left(p^{2}+r\right)(1-2 p)}{2 p-1} \\
& \times \cot ^{2}\left(\sqrt{\frac{1+\left(p^{2}+r\right)(1-2 p)}{2-4 p}}(x+y-2 p t)\right) \text {, } \\
& u_{9,10}= \pm \mathrm{e}^{\mathrm{i}(p x+q y+r t)} \sqrt{1+\left(p^{2}+r\right)(1-2 p)} \\
& \times \tan \left(\sqrt{\frac{1+\left(p^{2}+r\right)(1-2 p)}{2-4 p}}(x+y-2 p t)\right), \\
& v_{9,10}=\frac{1+\left(p^{2}+r\right)(1-2 p)}{2 p-1} \\
& \times \tan ^{2}\left(\sqrt{\frac{1+\left(p^{2}+r\right)(1-2 p)}{2-4 p}}(x+y-2 p t)\right) \text {. }
\end{aligned}
$$

\section{Conclusions}

We proposed a generalized tanh method for constructing a range of exact solutions for three well-known nonlinear complex coupled equations. Compared with the existing methods [26, 27], our method further exceeds their applicability in obtaining a series of exact travelling wave solutions. In this section, we compare with some existing results:

- The solution (21) with positive sign are equivalent to the solutions (4.21) and (48) in [26] and [27], respectively.

- The solution (23) with positive sign are equivalent to the solution (4.22) in [26]. 
- The solution (46) with positive sign are equivalent to the solutions (5.21) and (61) in [26] and [27], respectively.

- The solution (47) with positive sign are equivalent to the solution (5.22) in [26].

Therefore the solutions in [26] and [27] turn out to be special cases of the results from this paper.

Later, this paper will be studied further along. One avenue of additional investigation is the issue of conservation laws. The Lie symmetry analysis with double reduction will be implemented to dig out conserved densities and soliton solutions will lead to conserved quantities. Moreover, these three equations will be studied with fractional temporal evolution. This will lead to further generalized solutions that will be an addendum to the literature of NLEEs. These results will be reported soon.

\section{References}

[1] A.H. Bhrawy, M.A. Abdelkawy, Centr. Europ. J. Phys. 11, 518 (2013).

[2] A.H. Bhrawy, M.A. Abdelkawy, Indian J. Phys. 87, 665 (2013).

[3] E. Tohidi, A.H. Bhrawy, K. Erfani, Appl. Math. Model. 37, 4283 (2013).

[4] M. Eslami, B. Fathi Vajargah, M. Mirzazadeh, Ain Shams Eng. J. 5, 221 (2014).

[5] A.H. Bhrawy, Appl. Math. Comput. 222, 255 (2013).

[6] A.H. Bhrawy, M.A. Abdelkawy, A. Biswas, Commun. Nonlin. Sci. Num. Simul. 18, 915 (2013).

[7] M.A. Abdelkawy, A.H. Bhrawy, Indian J. Phys. 87, 555 (2013).

[8] A.H. Bhrawy, A. Yildirim, M.M. Tharwat, M.A. Abdelkawy, Indian J. Phys. 86, 1107 (2012).
[9] A.M. Wazwaz, Appl. Math. Comput. 200, 160 (2008).

[10] A.H. Khater, D.K. Callebaut, M.A. Abdelkawy, Phys. Plasmas 17, 122902 (2010).

[11] A.H. Khater, D.K. Callebaut, A.H. Bhrawy, M.A. Abdelkawy, J. Comput. Appl. Math. 242, 28 (2013).

[12] S. Ghosh, S. Nandy, Nucl. Phys. B 561, 451 (1999).

[13] R.A. Baldock, B.A. Robson, R.F. Barrett, Nucl. Phys. A 366, 270 (1981).

[14] A.N. Beavers Jr., E.D. Denman, Math. Biosci. 21, 143 (1974).

[15] Y.C. Hon, E.G. Fan, Nonlin. Anal. 71, 3501 (2009).

[16] X.B. Hu, B.L. Guo, H.W. Tam, J. Phys. Soc. Japan 72, 189 (2003).

[17] N.N. Rao, P.K. Shukla, Phys. Plasmas 4, 636 (1997).

[18] N.L. Shatashvili, N.N. Rao, Phys. Plasmas 6, 66 (1999).

[19] P. Saha, S. Banerjee, A.R. Chowdhury, Chaos Solit. Fract. 14, 145 (2002).

[20] A.R. Chowdhury, B. Dasgupta, N.N. Rao, Chaos Solit. Fract. 9, 1747 (1998).

[21] Y. Hase, J. Satsuma, J. Phys. Soc. Jpn. 57, 679 (1988).

[22] D. Rostamy, F. Zabihi, K. Karimi, S. Khalehoghli, Appl. Math. 2, 258 (2011).

[23] A. Maccari, J. Math. Phys. 37, 6207 (1996).

[24] H. Zhao, Chaos Solit. Fract. 36, 359 (2008).

[25] A.H. Bhrawy, M.A. Abdelkawy, A. Biswas, Optik Int. J. Light Electron Opt. 125, 1537 (2014).

[26] A. Bekir, Commun. Nonlinear Sci. Numer. Simul. 14, 1069 (2009).

[27] A. Jabbari, H. Kheiri, A. Bekir, Comput. Math. Appl. 62, 2177 (2011).

[28] B. Ahmed, A. Biswas, E.V. Krishnan, S. Kumar, Rom. Rep. Phys. 65, 1138 (2013). 